Hybrid Poly (hydroxy urethane) s; Folded Sheet Morphology and Thermo-

\title{
Reversible Adhesion
}

Anitha Sukumaran Nair. a, Suchithra Cherian ${ }^{b}$, Nisha Balachandran ${ }^{b}$, Unnikrishnan Gopalakrishna

Panickerc, Santhosh Kumar Kalamblayil Sankaranarayanan ${ }^{\text {a* }}$,

a Polymers and Special Chemicals Group, Vikram Sarabhai Space Centre

Thiruvananthapuram - 695022, India

${ }^{b}$ Analytical, Spectroscopy and Ceramics Group, Vikram Sarabhai Space Centre

Thiruvananthapuram - 695022, India

c Department of chemistry, National Institute of Technology, Calicut-673601

Email: santhoshkshankar@yahoo.com

\section{Supporting Information}

Figure S1 Chain structure of individual PHUs

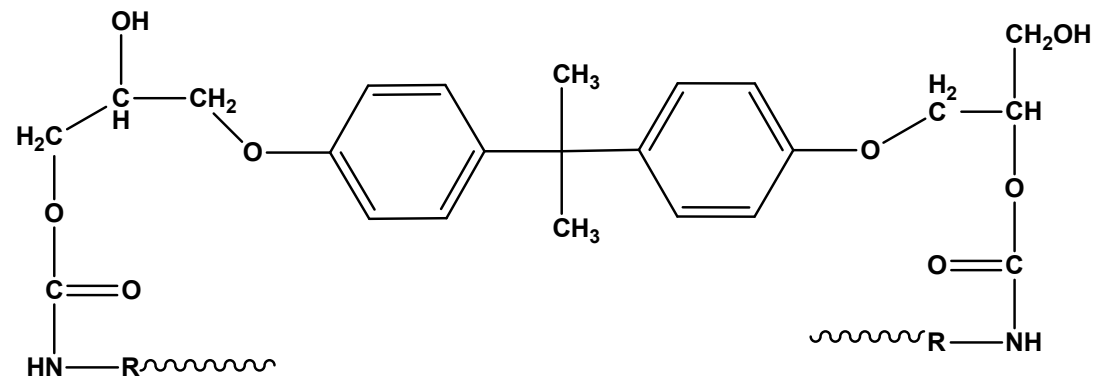

Chain structure of poly(hydroxy urethane) derived from BACC/PEA400 


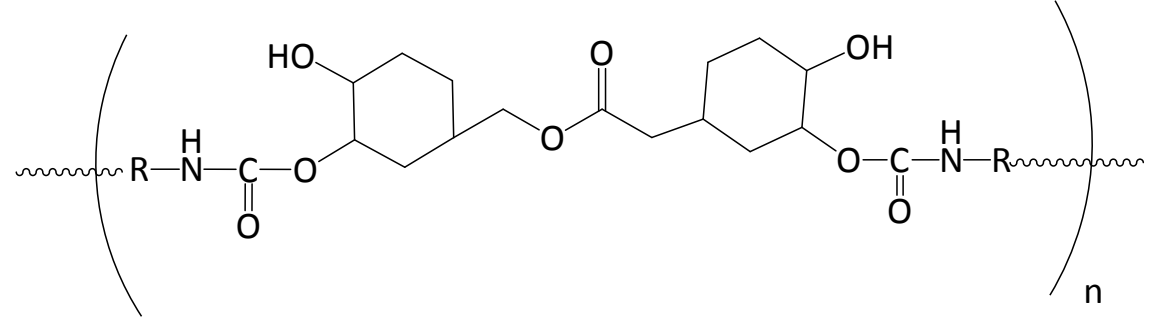

Chain structure of poly(hydroxy urethane) derived from CYCC/PEA400

Figure S2 DSC profile of homo and hybrid polyhydroxy urethanes.

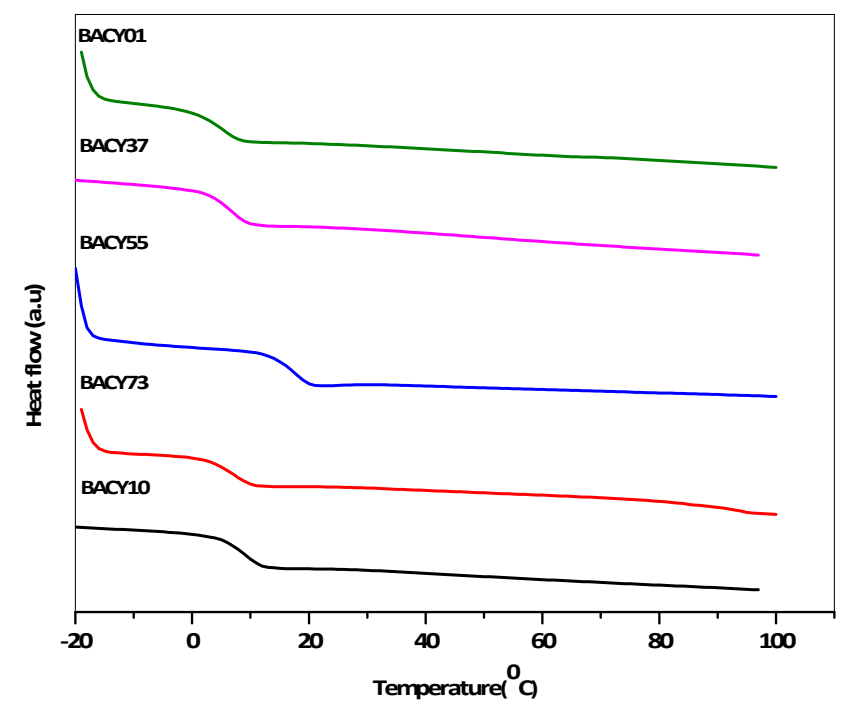

Figure S3 Softening point observed for different PHU's from differential scanning calorimetry (testing conditions: $5^{\circ} \mathrm{C} / \mathrm{min}$, under $\mathrm{N}_{2}$ atmosphere) 


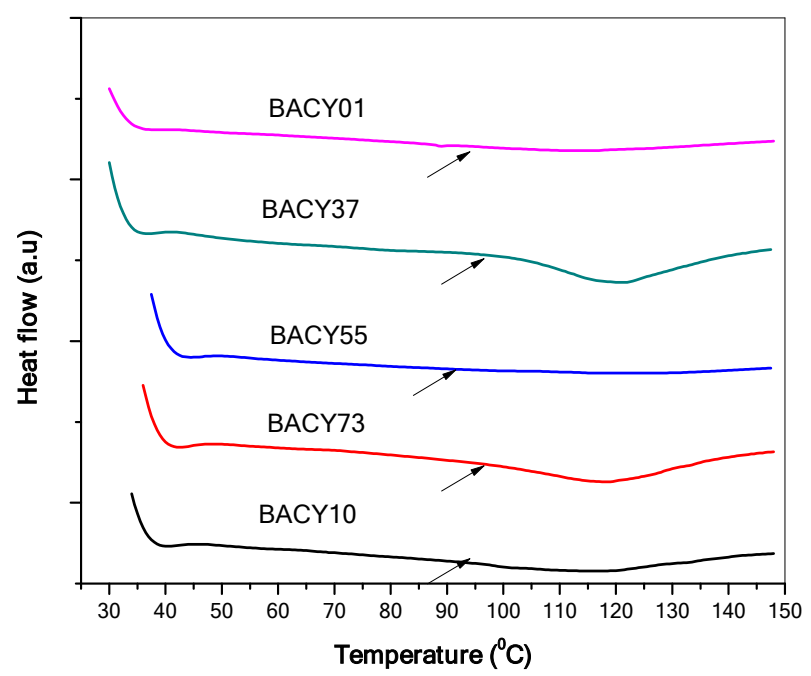

Figure S4 X-ray diffraction patterns of PHU's studied (representative cases are given)
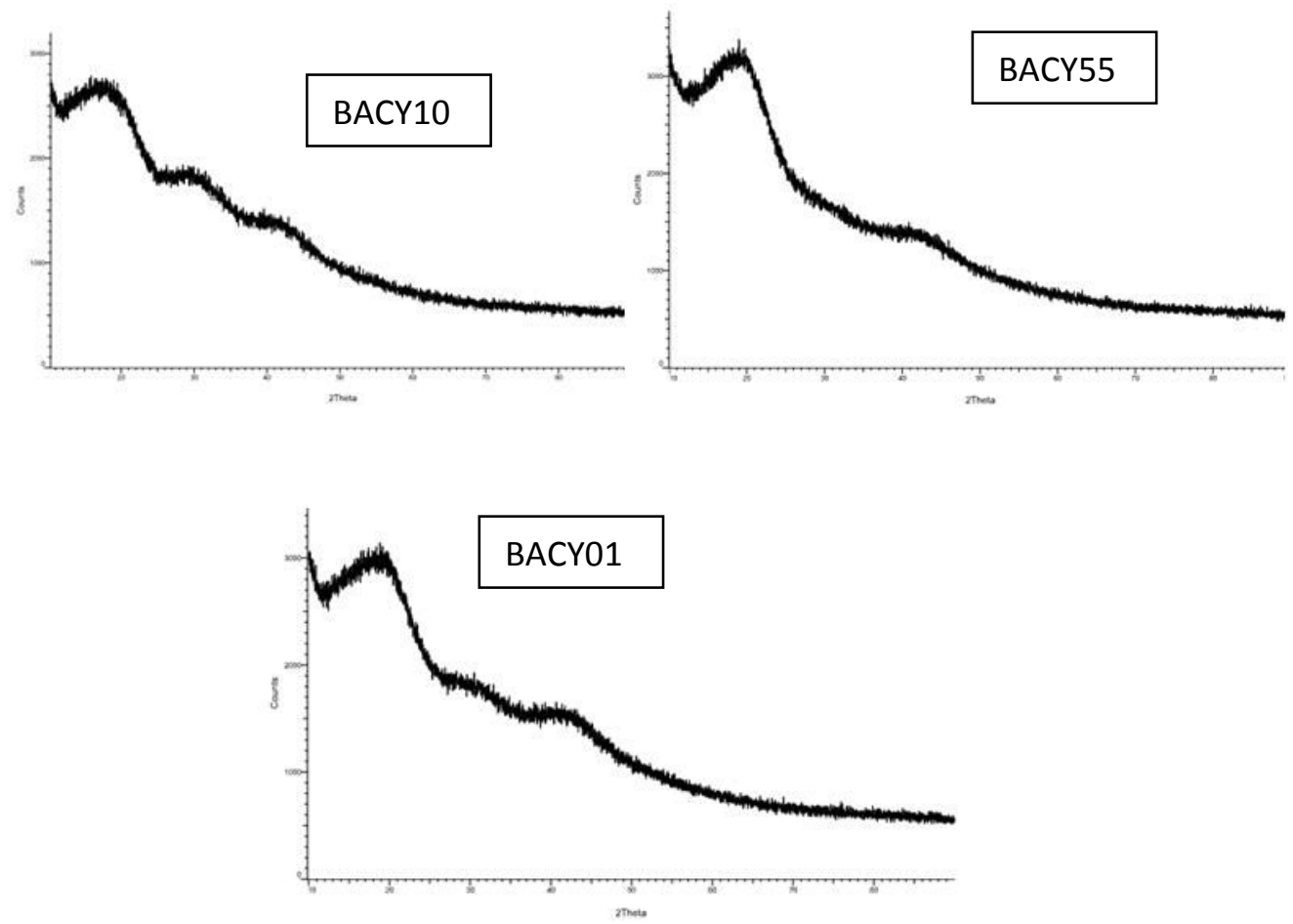
Figure S5 Temperature dependent FTIR spectra (normalized) of homo PHUs BACY 01 and BACY 10 in -CO regime and $-\mathrm{OH} / \mathrm{NH}$ regime at $30,100^{\circ} \mathrm{C}$ and cooled back to $30^{\circ} \mathrm{C}$.
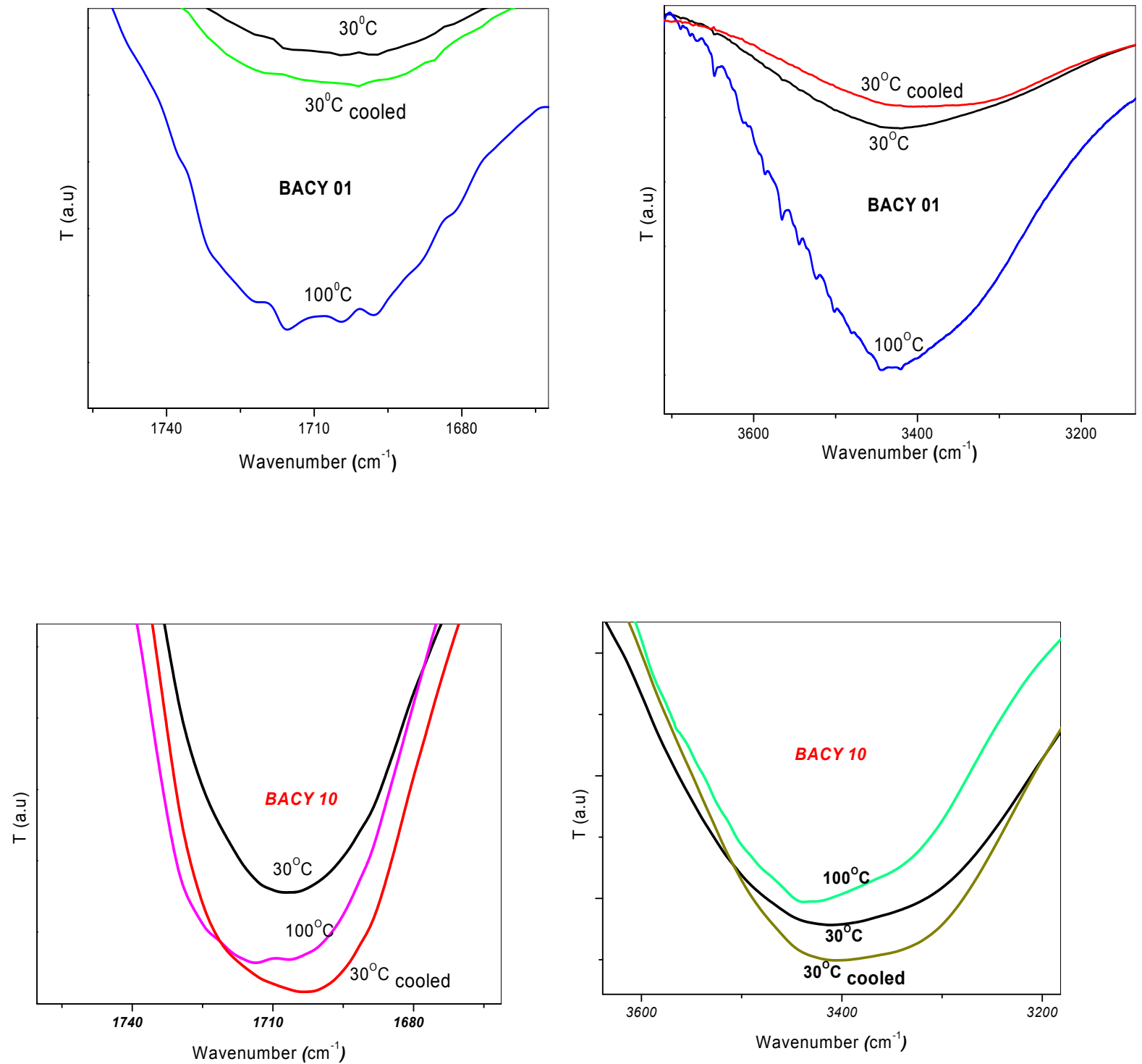
Figure S6 Change in complex viscosity with heating and cooling for BACY 55

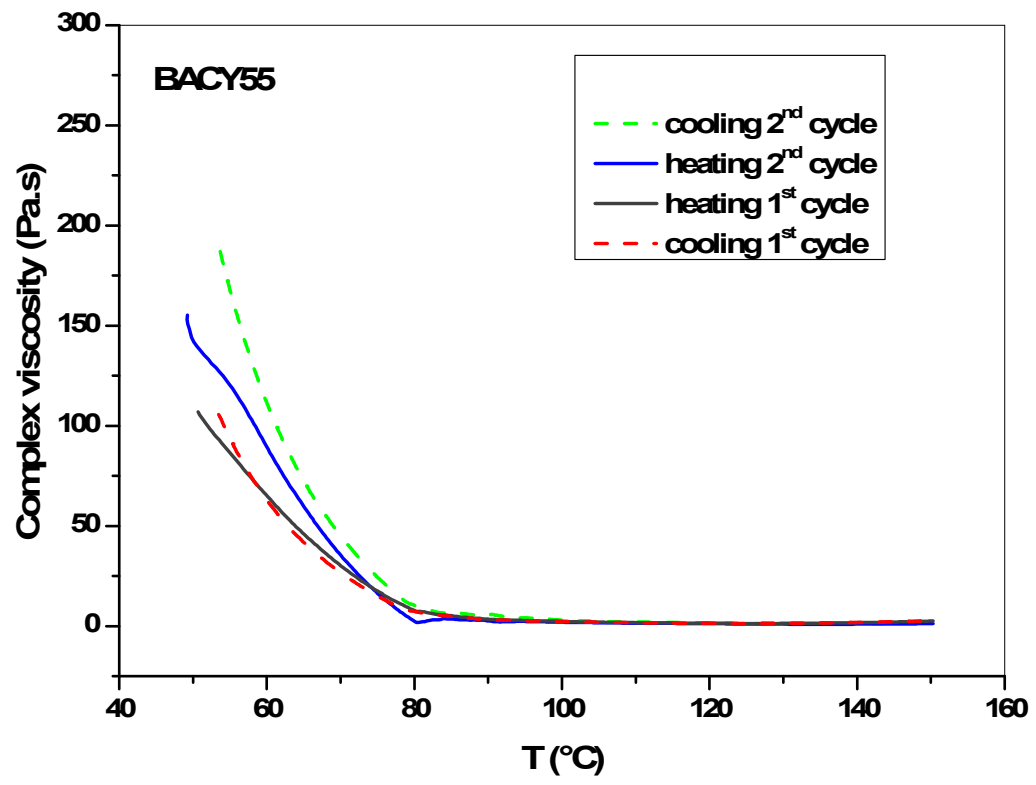

Figure S7 SEM images of PHU's focusing on the width of folded chains

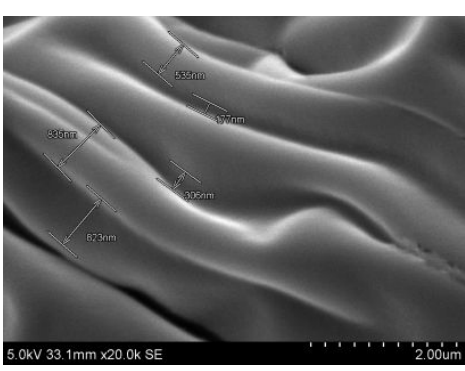

BACY10

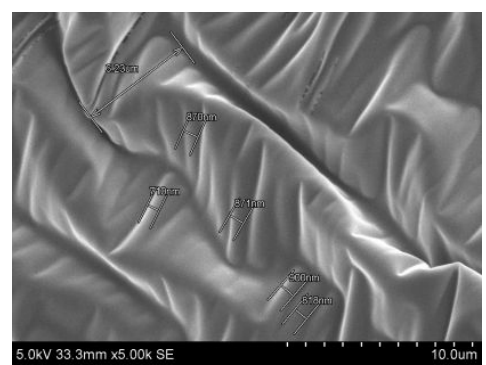

BACY73

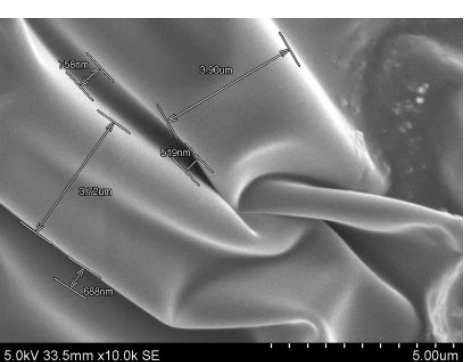

BACY 37 


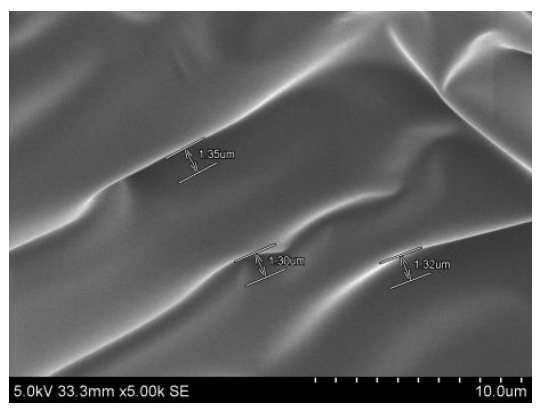

BACY01

Figure S8 A) Al specimen bonded with BACY 55 B) bonded specimen with hanging weight

C) heating the bonded area with hot air blower and D) debonded specimen at the inset.
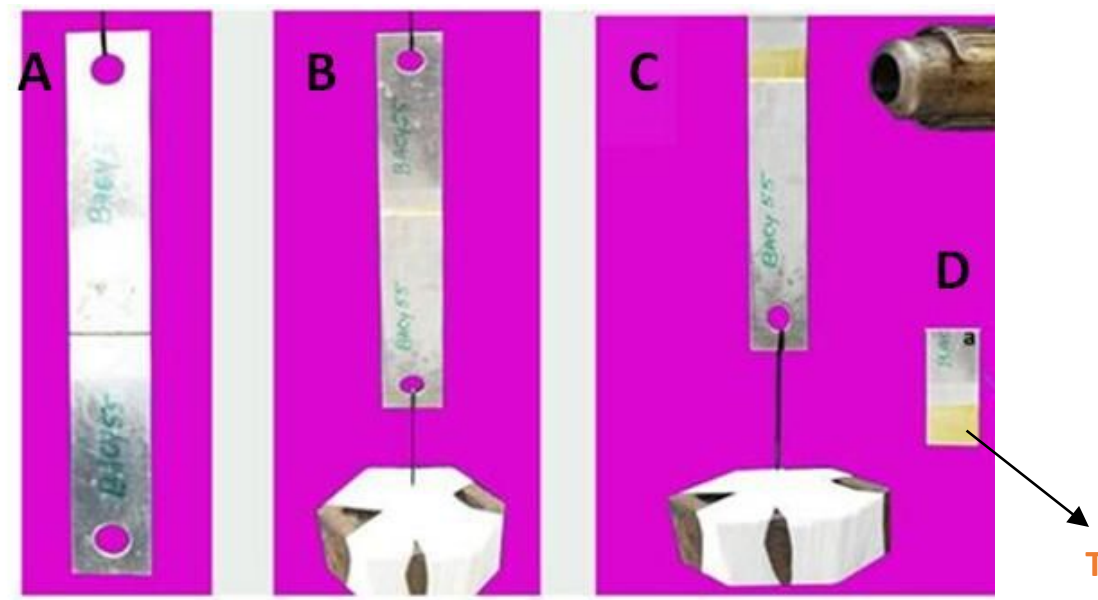

Thermally debonded PHU adhesive

Figure S9 Transparency of PHU's in the visible region obtained from solid state UV-visible spectroscopy. 


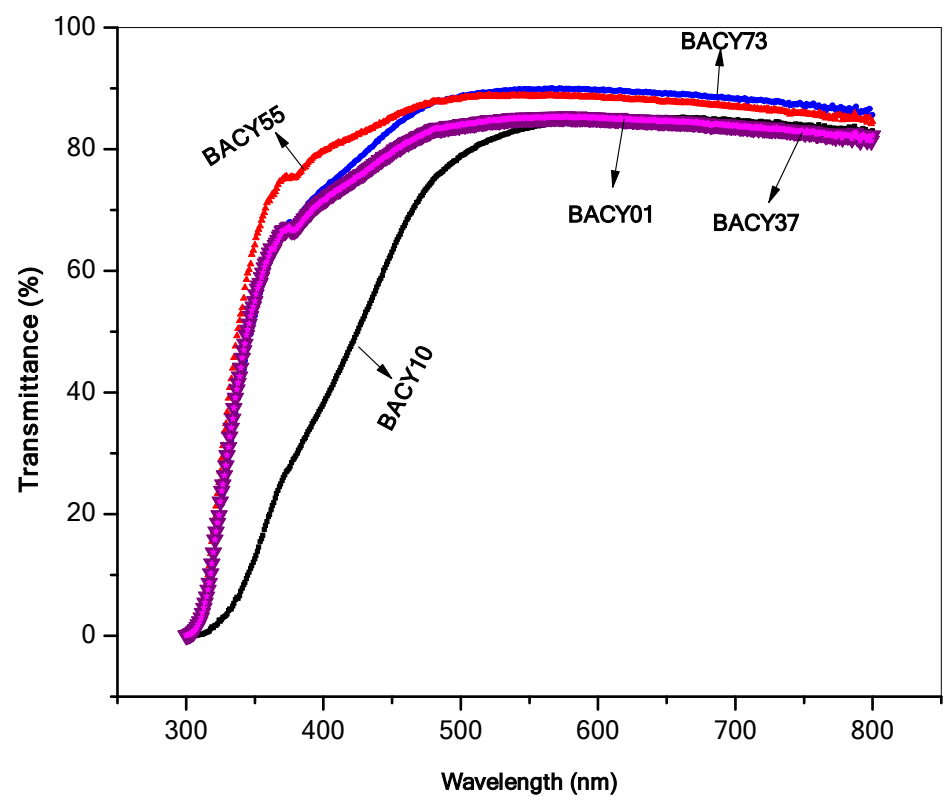

Figure S10 Thermogravimetric profiles of (polyhydroxy urethane) s exhibit good thermal stability.

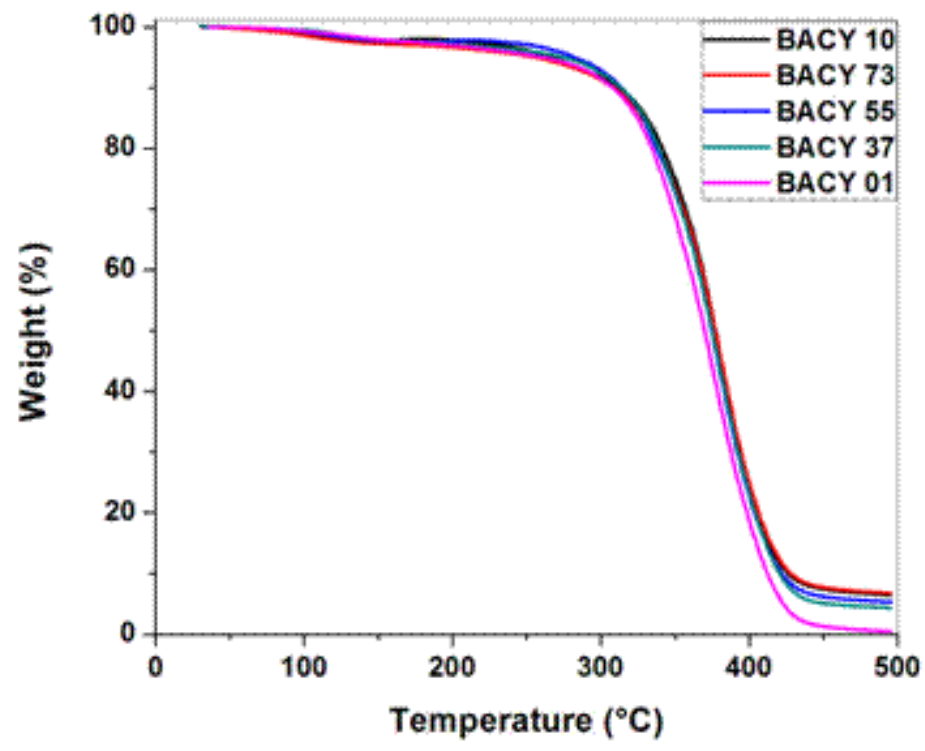

Figure S11 FTIR of BACC 


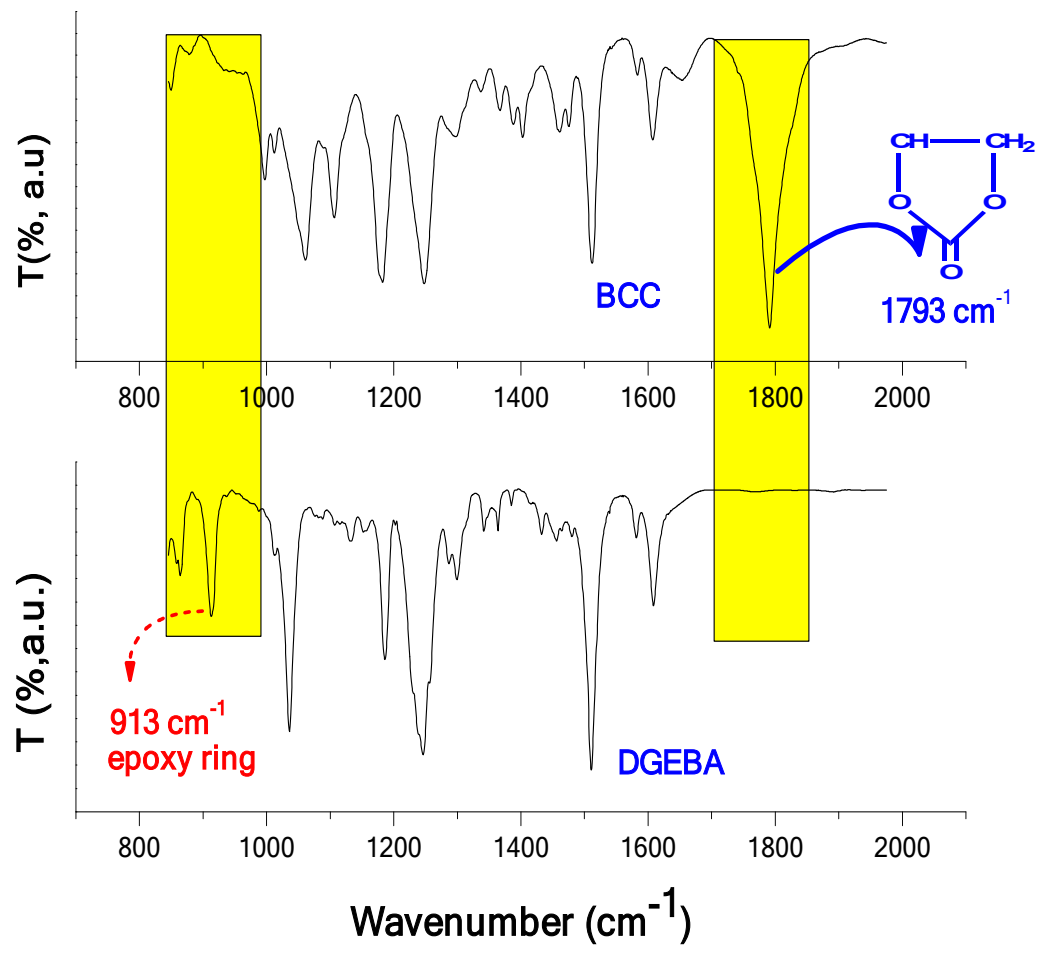

Figure S12 FTIR of CYCC

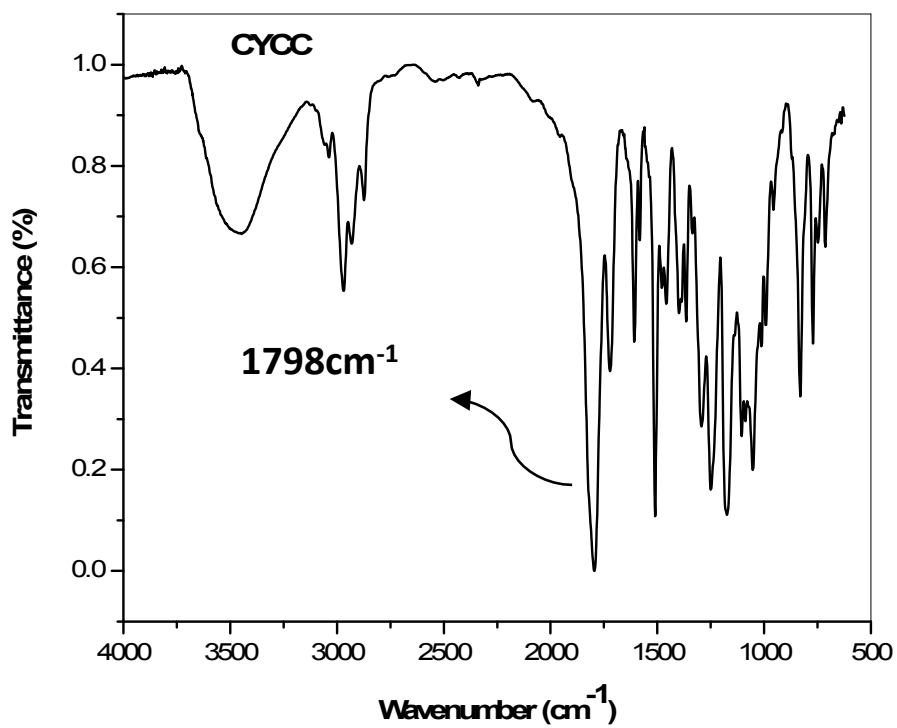


Table S1 Molecular weight of poly (hrdroxyurethane)s synthesized via CC-amine reaction (GPC method- THF as eluent)

\begin{tabular}{|l|c|c|c|c|c|c|}
\hline $\begin{array}{c}\text { Hybrid } \\
\text { networks }\end{array}$ & \multicolumn{3}{|c|}{ Protic } & \multicolumn{3}{c|}{ Aprotic } \\
\cline { 2 - 7 } & Water & Ethanol & Methanol & Acetone & EthylAcetate & DMF \\
\hline BACY 10 & - & + & + & + & + & + \\
\hline BACY 73 & - & + & + & + & + & + \\
\hline BACY 55 & - & + & + & + & + & + \\
\hline BACY 37 & - & + & + & + & + & + \\
\hline BACY 01 & - & + & + & + & + & + \\
\hline
\end{tabular}

Table S2 Solubility of hybrid PHUs in different protic and aprotic solvents

\begin{tabular}{|c|c|c|}
\hline Hybrid networks & Mn & Mw \\
\hline BACY 10 & 5300 & 11500 \\
\hline BACY 73 & 4600 & 10000 \\
\hline BACY 55 & 5100 & 10500 \\
\hline BACY 37 & 4900 & 96400 \\
\hline BACY 01 & 4600 & 9800 \\
\hline
\end{tabular}

+ Soluble $\quad$ - Insoluble 